\title{
Comparative Sequencing of ITS and D1/D2 Domains of Ribosomal DNA for Molecular Discrimination of Fungal Moulds
}

\author{
R. Baskar, K. Umamaheswari
}

Department of Biotechnology, University of Madras, Chennai, India- 600025.

BACKGROUND: Diagnosis of mycotic diseases in critically ill patients is challenging because current diagnostic methods lack sensitivity and specificity. Advances in molecular technology show great potential for the rapid detection of fungal pathogens and several potentially discriminating, species-specific targets within the fungal genome have been developed. Ribosomal targets, especially the ITS and D1-D2 regions have shown particular promise for the molecular discrimination of fungal pathogens. In the present study, we assessed the utility of ITS and D1/D2 domain as amplification targets for molecular discrimination of clinically relevant fungal moulds.

MATERIALS AND METHODS: A total of 80 fungal species previously isolated from clinical specimens collected from patients with Cancer and Pulmonary Tuberculosis were included in this study. All the fungal isolates were presumptively identified based on their macro- and micro-morphological characteristics. The fungal DNA was extracted using standard liquid nitrogen protocol and the ITS and D1/D2 domains of Ribosomal DNA were amplified using ITS1/ITS4 and D1/D2 primers. The amplicons were purified, sequenced and the sequences were analyzed with the curated molecular database and assigned to the reference isolate sequences with the highest bit score for species confirmation.

RESULTS: All the 80 isolates were preliminarily identified and speciated based on phenotypic characteristics. The ITS and D1/D2 domains of the ribosomal DNA were successfully amplified for species discrimination using fungus-specific universal primer ITS1/ITS4 and D1/D2 respectively. The species were confirmed as Aspergillus (A. fumigatus, A. flavus, $A$. niger, $A$. terrus, $A$. nidulans, $A$. ocharceus, $A$. versicolor) Mucormycetes ( $R$. microsporus, S. racemosum, L. corymbifera, L. ramosa, L. ornata C. bertholletiae), Penicillium ( $P$. oxalicum, $P$. chrysogenum, $P$. citrinum, P. purpurogenum), Hyalohyphomycetes (Fusarium solani, Paecilomyces lilacinus, Trametes polyzona, Porostereum spadiceum, Coprinopsis cinerea, Hormographiella aspergillata), Dermatophytes ( $T$. mentagrophytes, $T$. interdigitale, $T$. rubrum, $M$. gypseum), Phaeohyphomycetes (Alternaria alternata Curvularia Iunata, Curvularia verruculosa) and yeast like fungi (Trichosporon asahii, Geotrichum candidum and Rhodotorula mucilaginosa). The primers targeting ITS domain was found to be more reliable in discriminating the species of Aspergillus, Penicillium and Dermatophytes while D1/D2 domain were reliable in discriminating Mucormycetes, Hyalohyphomycetes and Phaeohyphomycetes.

CONCLUSION: Sequencing of the ribosomal genes has emerged as a useful diagnostic tool for the rapid identification of pathogenic fungi. In the present study, the utility of the ITS and D1/D2 domain of the ribosomal DNA sequencing was analyzed for fungal species discrimination. Both the domains could identify fungal species, while ITS region was more reliable in discrimination of majority of the species under different genera. Species level identification has become mandatory with incidences of antifungal resistance. Hence Comparative Sequence analysis of these two domains on larger number of fungal species would help us to choose an appropriate targets for species level identification.
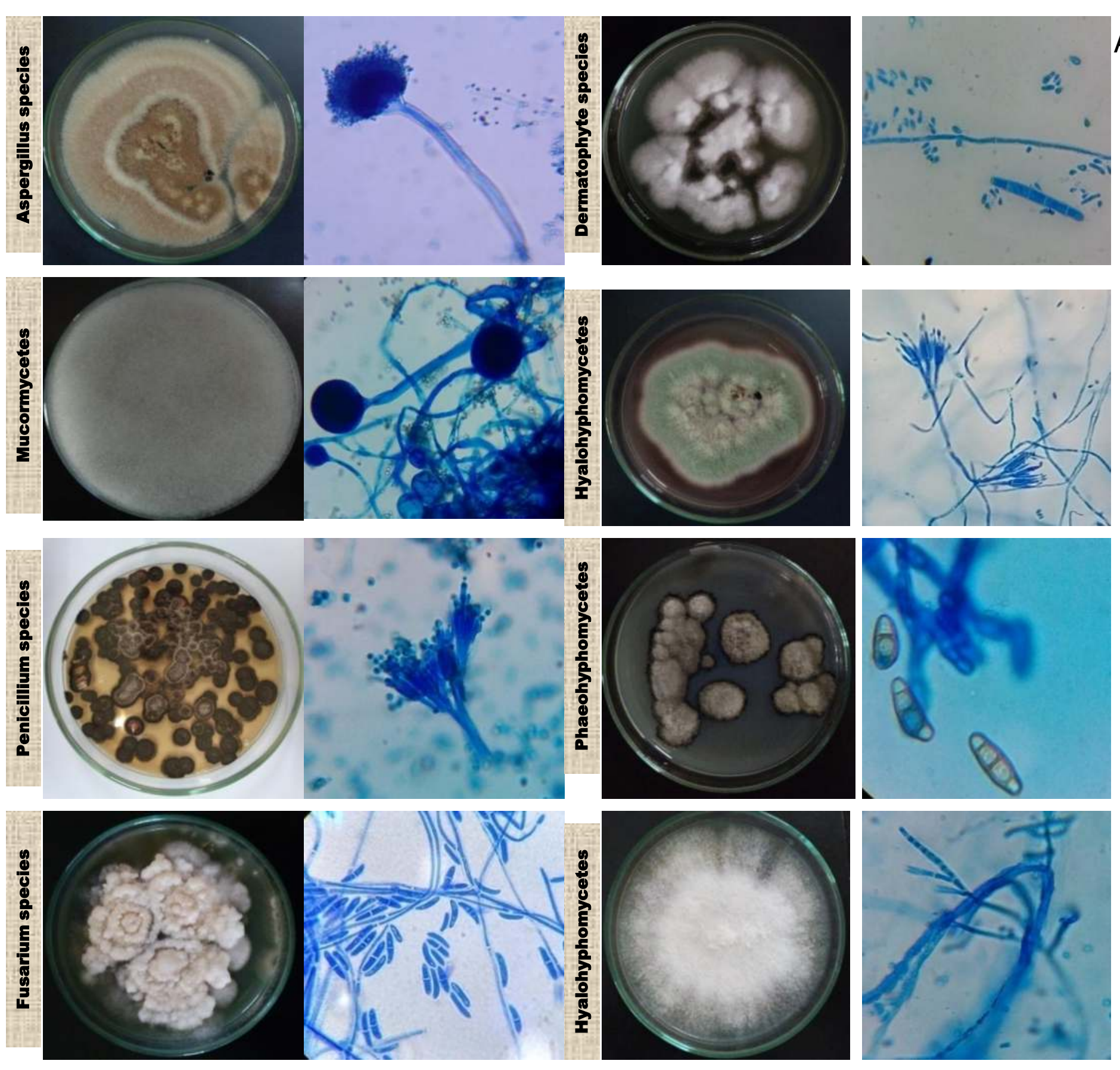
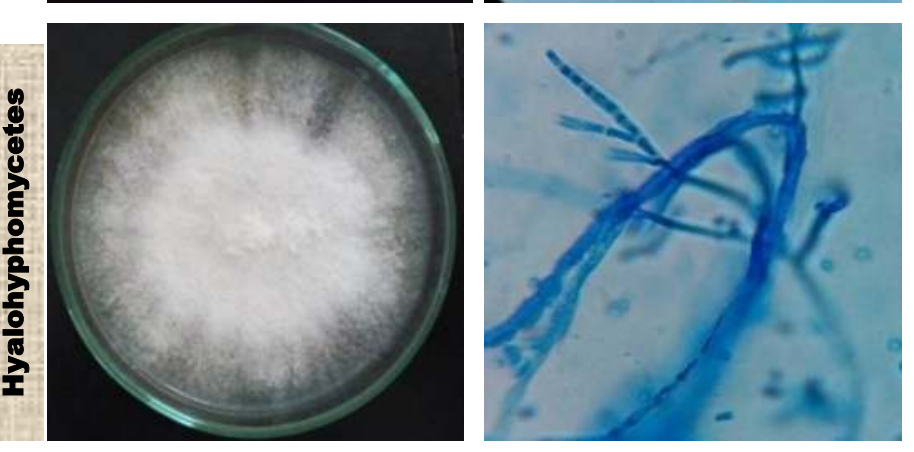

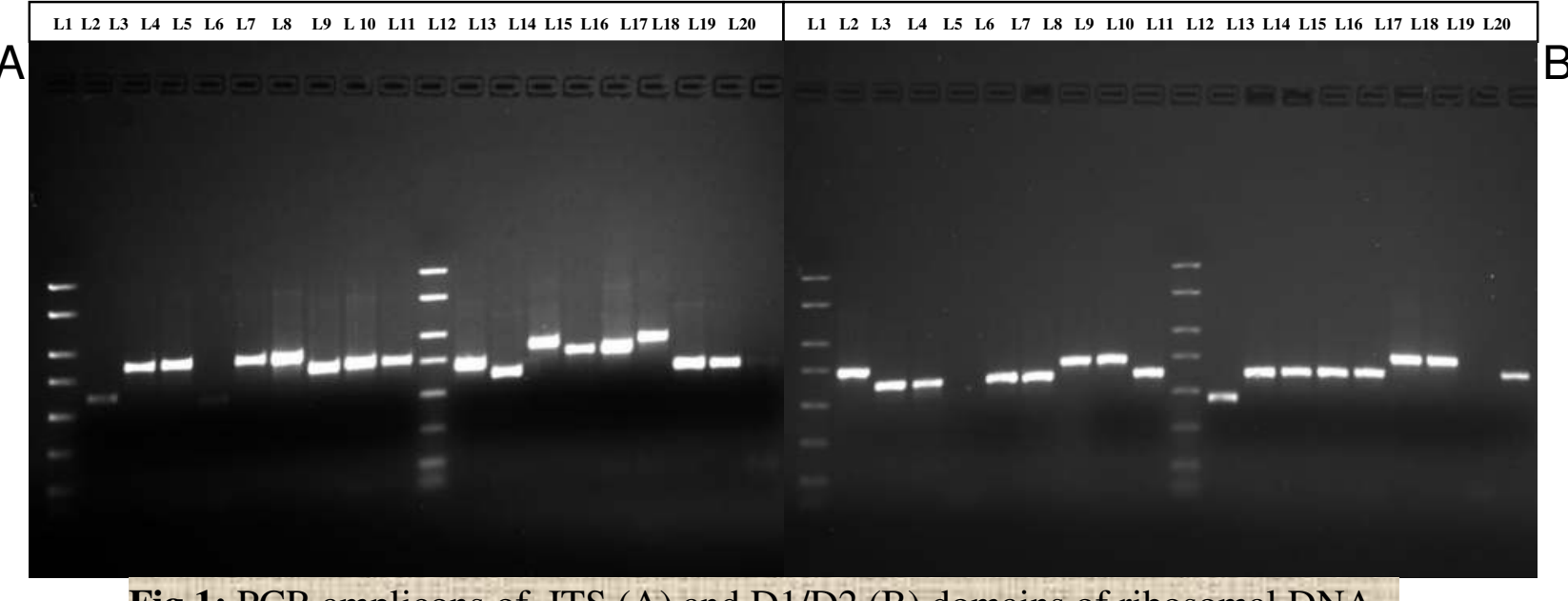

Fig 1: PCR amplicons of ITS (A) and D1/D2 (B) domains of ribosomal DNA

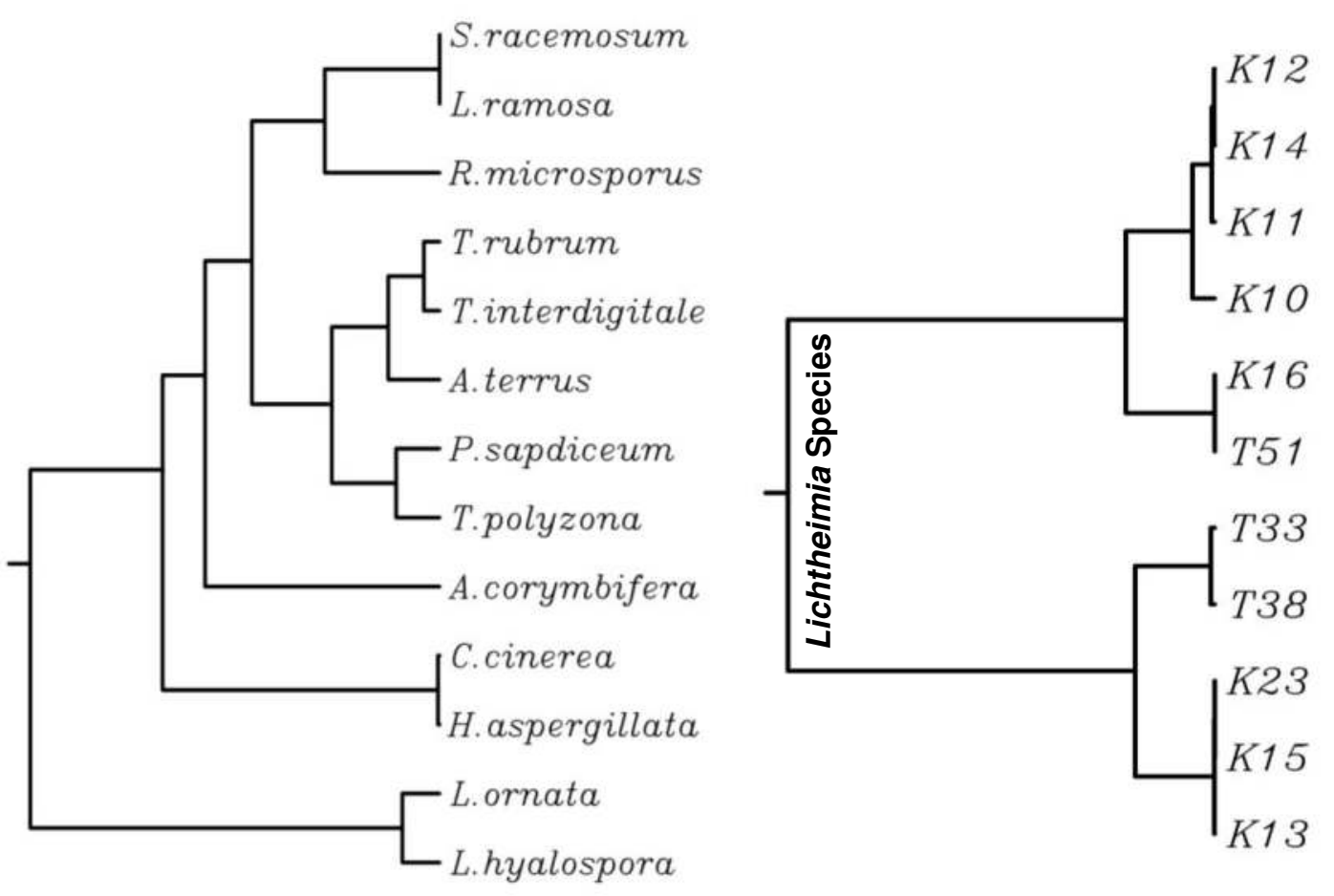

Fig 2: Phylogram of Fungal species (ITS sequencing- UPGMA) 